solutions of the sodium and ammonium picrates show parallel intensities of color, they diverge rapidly at $100^{\circ}$. This may be accounted for either on the basis of a decomposition of the ammonium salt by heat or on the basis that the sodium and ammonium salts may possess different transition-concentrations at the higher temperatures. Furthermore, it is not clear why the heated ammonium picrate series should show less intense colors at $100^{\circ}$ than the aqueous solutions. This phenomena, the progressive influence of heat on picrates and o-nitro phenolates, and other studies of the latter will be made.

\title{
Summary.
}

I. Color changes of picrate solutions are produced by changes of concentration, temperature and alkalinity.

2. Color ratios were measured and correlated in curves.

3. The order of reagents and conditions increasing the color are: $\mathrm{HCl}$, 100\% alcohol, $95 \%$ alcohol, alkalies and heat.

4. Picric acid probably exists in two isomeric forms; changes of color are explained on the basis of disturbance of its benzoid-quinoid equilibrium.

5. Deviations from Beers' law are also quite satisfactorily accounted for.

SEATTLE, WAsB.

CONTribution from the Chemical Laboratory of the UNIVHRstTy OF WashINGTON.]

\section{FALLACIES IN COLORIMETRY.}

By WrLLIAM M. DEHN.

Received February 27, 1917.

In colorimetric experiments certain factors introducing variations, and errors $^{1}$ are frequently overlooked-chief among these are variable sensitiveness $^{2}$ of vision to different concentrations and the bicolored nature of the solution.

In most colorimetric determinations, ${ }^{3}$ the depths of solutions of the color standards are matched by various depths and concentrations of the solutions to be estimated; usually we have two columns of solutions of unequal length whose tints are matched to the limit of sensitiveness of the eye and whose solute molecules are assumed to be the same in number. That the solute-molecules are not necessarily the same, not only in number but in composition, may be concluded from studies of ( $\mathrm{I}$ ) their different

i For a discussion of colorimetric methods, see Turs JouRnAL, 27, i I 22.

2 Horn, Am. Chem. J., 35, 253; Horn and Blake, Ibid., 36, I95, 5 I6. These authors discuss variable sensitiveness in colorimetry. They show that, with equal depths "at certain definite concentrations, the comparisons in colorimetric determination of chromium can be made with greater ease and accuracy than at other concentrations," and it is held by them that this reiation is "perfectly general throughout colorimetry."

${ }^{3}$ Scores of colorimeters varying from simple vials and test tubes to complicated 
optical distributions; (2) different equilibria resulting from ionization; (3) different equilibria resulting from hydrolysis and, (4) different equilibria resulting from chromoisomerization.

\section{Different Optical Distributions.}

In respect to different optical distributions, four cases appear-the matched solutions may have:

(I) Equal volumes, equal depths, and equal cross sections.

(2) Equal volumes, equal depths, and unequal cross sections.

(3) Equal volumes, unequal depths, and unequal cross sections.

(4) Unequal volumes, unequal depths, and unequal cross sections.

It will be shown that all of these geometrical conditions involve errors which increase from Case $I$ to Case 4.

Case I.-If, for instance, two cylinders of equal diameter are used and the solutions contained therein are viewed along the lines of the major contrivances possessing optical and mechanical accuracy are described in the literature. The following bibliography is arranged largely in chronological order:

Dingler's polytech. J.: Rheineck, 201, 467 (1871); Salleron, 203, 141 (1872); Stammer, 203, $x_{37}$ (1872); Bremmer, 205, 278 (1874); Günsberg, 228, 457 (1878).

Chem. News: King, 3I, 133 (1875); Leeds, 37, 229 (1878); Bottomley, 38, I9I (1878); 39, 276 (1879); Crooks, Olding and Tidy, 45, 170 (1882).

J. Chem. Soc.: Herepath, 5, 27 (1853); Miller, I8, I 18 (1865). (1913).

Z. anal. Chem.: Meisling, 43, 137 (1904); Autenrieth and Königsberger, 52, 137

Chem. Ztg.: Scholz, 38, 487 (1913); Günther, 33, 318 (1909); White, 36, 747 (1912); Klemperer and Löwe, 36, 853 (1912).

THIS Jotrnal: Schreiner, 25, 1058 (1903); 27, II92 (1905); Smeaton, 28, 1433 (1906); Stokes and Cain, 29, II4 (I907); Steiger, 30, 215 (1908); Frankforter, Walker and Withoit, 3I, 35 (I909); Campbell and Hurley, 33, III2 (IgII); White, 34, 654 (IgI2).

Pusch, Arch. Pharm., [3] 14, 227 (1879); Giannetti, Gazz. chim. ital., 16, 65 (1886); Krüss, Z. anorg. Chem., 5, 325 (I894); Lovibond, J. Soc. Chem. Ind., 9, 1o (I900); Sanger and Shephard, Bull. Am. Inst. Mining Eng., II, 743 (I906); Eijkman, Proc. Acad. Wetens., 8, 166 (1905); Ivey, J. Frank. Inst., 164, 47, 42 I (1907); Paravicini, Stahl u. Eisen, 29, I233 (Igog); Roberts, Bull. Hyg. Lab. U. S. P. H. Serv., 66, 3 (19ro); Lovibond, Pottery-Gazz., 35, I 269 (I91 I); Bobier, Bull. soc. pharm. Bordeaux, 50, 480 (1911); von Fellenberg, Mitt. Lebensm. Hyg., I, 35I (I91 I); Nuttig, J. Wash. Acad. Sci., 2, I83 (I9r2); Grützner, Arch. ges. Physiol., I44, 545 (1912); Toggenburg, Schweiz. Wochsch., 50, 417 (1912); White, $Z$. angew. Chem., 25, 1563 (1912); Autenrieth and Königsberger, Münch. med. Woch., 57, 998 (1910); Silber, Z. nahr. Genussm., 26, 282 (I9I3); Scheaff, J. Am. Med. Assoc., 65, 18I (Igro); Verbeek, Z. angew. Chem., Aufsatz, 27, 203 (rgrr); Stanford, Z. physiol. Chem., 87, I59 (1913); Whitson, Bull. Wis. Agr. Exp. Sta., 85 .

U. S. Pat.: White, 840,538; Parks, 977,964; Lovibond, 987,I48; Hellige, 998,09r.

Ger. Pat.: Scezepanik, 191,738; Plesch, 223,183; Field and Schlesinger, 235,541.

Eng. Pat.: Scezepanik, 22,995; Sanger and Shephard, 23,429.

Other names associated with colorimeters, but whose bibliographies have not been traced are: Gallenkamp, Hehner, Holtz, Huber, Koenig, Kober-Lenzmann, Mayer, Proskauer, Schmidt-Haensch, Duboscq, Kennicott-Sargent. 
axes, such solutions appear as truncated cones whose distant cross sections are concentric with the near and apparently larger cross section. That these identical figures may appear identical, they must be viewed at equal distances and the lines of the major axes must always intersect on the retina. ${ }^{1}$ When these conditions are met with the viewed fields are identical but never homogeneous, owing to the fact that the near and distant surfaces of the liquids intercept unequal lines which continue and intersect on the retina. Therefore, though the central portions ${ }^{2}$ of the fields may be nearly homogeneous, the areas between the circumferences of the two concentric circles present fields of color rapidly decreasing toward the outer edges. The nearer the eye is to the viewed cylinders of solution, the less homogeneous will the viewed fields become; only at great distances will the viewed volumes appear approximately homogeneous and identical.

In a similar manner it may be shown that other forms of equal volume, equal depth and uniform cross section involve the same errors. Never are the fields of color homogeneous, for the reason that the near and distant bounding surfaces are parallel, whereas the true lines of vision may be conceived to radiate from the retina and become intercepted between the nearer and distant parallel planes, hence are of unequal length.

Case 2.-With forms involving equal volumes, equal depths and different cross sections, as, for instance, with cones, pyramids, sectors of spheres and their truncated forms, it can be demonstrated in a similar manner that homogeneous fields cannot be obtained; only with frustrums of hollow spheres can homogeneity ${ }^{3}$ be obtained and then only when the points of vision are coincidental with the spherical centres. However, no colorimeter with containing vessels of the sphere-frustrum form has been constructed, therefore no existing differential colorimeter is free from errors of optical distribution.

1 Of course, the assumption is made that the eye operates as a point of vision, whereas owing to measurable widths of pupil and retina, the eye presents an area of vision. This condition tends to compensate the above-described variations.

2 Some colorimeters are so constructed as to bring into juxtaposition the central portions of the two fields and thus to reduce to a minimum this possibility of error.

s Both homogeneity and identity of color are possible only when equal concentrations of solute molecules are contained in equal volumes of the sphere-frustrum form. Even then other constant conditions are necessary, such as concentration of $\mathrm{OH}^{-}$and $\mathrm{H}^{+}$, temperature and intensity of illumination.

When square uniform bottles of clear glass as containing vessels are viewed at distances of many feet, the sphere-frustrum formed is approximated. See THIs JouRNaL, $36,407,837$ (1914). This simple colorimeter method has in fact many advantages over costly and complicated colorimeters.

For the absorption of certain aniline dyes by the glass of containing vessels, see Roberts, Bull. Hyg. Lab. P. H. and M. H. Serv., 66, 3. 
Case 3.-When equal volumes of unequal ${ }^{1}$ depth are viewed longitudinally, the near cross sections being the same or different, not only are homogeneity and identity of color impossible for above-mentioned reasons, but two other physical conditions producing color variations are introduced.

First, the more distant solute-molecules conceivably vary in size inversely as the square of the distance from the point of vision. Though, of course, no individual molecule is visible, it is possible, though not demonstrable, that in near and distant viewings there may be physical differences produced in this manner by individual molecules or by molecules en masse. It is very probable, for this reason and for reasons given above, that the homogeneity of the viewed field is disturbed by the use of long tubes. Hence, it cannot be held that different columns of liquids of the same apparent tint contain the same number of solute-molecules.

The second physical conditions producing color variations in equal volumes of unequal depths is that more solute-molecules ${ }^{2}$ reill constantly be behind each other along the lines of vision in the longer columns than in the shorter columns. This physical difference probably affects the absorption of light like colored, transparent disks of glass on a white field, when viewed singly or in columns of varied depths. The latter positions give darker shades of color, whereas the former, within certain limits of being loosely or densely crowded, give the same shade. Therefore, it is not to be expected that a solution of definite concentration viewed in a certain depth will have exactly the same shade of color as that, for instance, of a solution in double concentration and viewed in one-half of its depth.

1 Colorimeters of the immersion type involve volumes of liquids viewed as truncated cones. They approximate the sphere-frustrum form, when the depths of colored solutions are small and the distances from the eye are comparatively large. However, under any condition, the viewed fields are not homogeneous, the outer areas being darker than the central areas.

Furthermore, in colorimeters of this type, the total viewed volumes are not directly proportional to the movements of the near, trans-base bounding surfaces. If the eye is at a distance of $a$ from this nearer surface and at a distance of $a+b$ from the further surface, when the former, with a radius of $r$, is moved toward the latter a distance of $c$, the ratio of viewed volumes can be shown to be expressed by

$$
\frac{a^{2}\left[(a+b)^{8}-(a+c)^{2}\right]}{(a+c)^{2}\left[(a+b)^{3}-a^{3}\right]}
$$

and not expressed by the ratio of $b . c / b$. When, for instance, $a=2, b=2$ and $c=1$, the ratio of volumes is not I to 0.50 but $I$ to $0.29+$. Now, then, if matchings of color of the entire fields are made, in this case, it will be observed an error of $42 \%$ is made.

${ }^{2}$ Of course, it may be argued that the diameters of molecules are insignificant when compared to the widths of intermolecular spaces and that consequently this physical influence is nil. Though perhaps nil in dilute solutions, packing of solute molecules in concentrated solutions certainly produces color variations, and possibly this is the most important cause of lack of sensitiveness to changes of concentration in deep columns or in solutions already very concentrated. 
Case 4.-When unequal volumes in unequal depth and different cross sections are viewed in colorimeters, there are introduced not only all possible errors of different optical distributions, and of the two possible physical effects mentioned, but also disturbances of the three equilibria of ionization, hydrolysis, and isomerization.

\section{Disturbances of Equilibria Resulting from Ionization.}

In considering the effects of ionization on colored solutes, two conditions must be recognized, viz., (I) the ionization of the solute molecules itself, and (2) the ions of the solution as affecting the solute molecules. For example, on the one hand, the ions yielded by methyl orange must be considered, and, on the other hand, the ions of acids and alkalies affecting methyl orange molecules must be considered.

Opinion is almost universal that the one, the other or both types of ionization affect the color of solute molecules. However, studies ${ }^{1}$ on the mass action of water on dyes, and especially on methyl orange, clearly demonstrate that the cause of color changes are primarily, if not entirely, independent of both types of ionization. In fact, it has been found that the molecular states of colored solutes are largely affected by hydrolysis and this influence, rather than the degrees of ionization, is operative in transforming the molecules and in producing color changes. Therefore, whether or not ionization figures at all in color changes, it is certain that other factors, such as the stability of the salts of the indicator, the mass influence of water, and the effects of temperature are far more important. ${ }^{2}$

Disturbances of Equilibria Resulting from Hydrolysis.

Whereas, in colorimetry the solutions estimated as well as the solutions used for comparison are almost universally assumed to be monochromatic, experiments show that many of them are dichromatic, ${ }^{3}$ that is, they are usually bicolored, like materials used as indicators.

During the past three years, experiments have been carried on in this laboratory to estimate the variations of tints of colored solutes when affected by ( $\mathrm{I}$ ) acids and alkalies, (2) heat and cold, and (3) dilution and concentration.

General knowledge in respect to the action of acids and alkalies on bicolored compounds is extensive and need not be discussed here. This important observation must be made-at sufficiently great dilution, many, if not all, bicolored substances, whether in acidic or in alkaline solutions, are

I See papers preceding.

2 For the effects of temperature on color, see especially Kurbatov, J. Russ. Phys. Chem. Soc., 39, 456 (1909); see also This Journal, 36, 845 (19r4).

8 Some may even be considered to be polychromatic. Many dyes, giving progressive changes of color from strong acid concentrations to strong alkaline concentrations, or vice versa, can be showed to be mixtures of only two forms. 
of the same tint. Therefore, with indicators, not only are the equilibrium actions of acids and alkalies to be considered but also the mass action of water; in other words, it is not a matter of indifference in colorimetry what the concentrations are of the solutions used. This was pointed out by Horn ${ }^{1}$ and others, and demonstrated in an earlier ${ }^{2}$ and the preceding papers.

It was showed first that all solutions of chromates and dichromates above $0.007 \%$ concentrations are mixtures of the two in aqueous equilibria. In the preceding paper it was showed that solutions of picrates above $0.001 \%$ concentrations are mixtures of tautomeric forms in aqueous equilibria. In a preceding paper it was showed that many dyes, especially dyes used as indicators, are changed in color by the mass action of water. Finally it was demonstrated that all aqueous solutions of methyl orange, whether acidic or alkaline in reaction, are chromoisomeric mixtures in aqueous equilibria.

In all colorimetric methods, it is either stated or implied that Beers' $1 \mathrm{aw}^{3}$ is valid, namely, that the tint of colored solutions is dependent upon the mass of the solute and is independent of dilution. That this law does not hold in many cases, as recognized by numerous investigators, ${ }^{4}$ is easily understood, when it is observed that water and other solvents have a transforming influence on many colored solutes. Therefore, in all colorimetric methods such influences must be recognized, the limits of dilution ${ }^{5}$ must be set and comparisons must be made under identical conditions.

\section{Disturbances Resulting from Chromoisomerization.}

Bicolored dyes, as methyl orange, undergo tautomeric changes when subjected to the influences of (I) acids and alkalies, (2) heat and cold, and (3) dilution and concentration. These influences have been discussed sufficiently in the foregoing. It needs only to be pointed out that such chromoisomerizations may be disturbed in the presence of constant concentrations of acids, alkalies and solvent, through changes of temperature. Therefore, constant temperatures, as well as constant concentrations, must be observed in all colorimetric methods.

1 Loc. cit.

2 This Journal, 36, 329 (1914).

Ann. Physik, [2] 86, 78 (1852).

- See especially Hantzsch's and Horn's papers. Also Picard, Ann., 381, 347 (1913); Stewart and Wright, Ber., 44, 2819 (I9I I),

5 "It is either stated or implied in descriptions of colorimetric methods that they are to be applied to dilute solutions. This practice seems to have originated in the observation that the results of parallel determinations made in deeply colored (not very dilute) solutions do not approximate sufficiently to the average value." Horn, Am. Chem. J., 35, 253 (1906). 


\section{General Conclusions.}

Conclusions drawn from the above may be illustrated in connection with a favorite colorimetric method, namely Folin's creatinine estimation, ${ }^{1}$ involving the reducing effect of creatinine on a $\mathrm{NaOH}$ solution of picric acid. Possible errors are as follows:

I. Mechanical ${ }^{2}$ errors of the colorimeter.

2. Optical errors of the colorimeter, as discussed above.

3. Optical errors resulting from varied light. ${ }^{3}$

4. Errors resulting from volumetric readings.

5. Errors of dilution, ${ }^{4}$ as discussed above.

6. Errors resulting from varied temperatures. ${ }^{5}$

7. Errors resulting from varied times ${ }^{6}$ of standing.

8. Errors resulting from varied quantities of $\mathrm{NaOH}^{7}$ used.

9. Errors resulting from varied quantities of picric acid 8 used.

Io. Errors resulting from other substances present in the urine, in the meat extract, etc., such as proteins, ${ }^{8}$ urinary pigments, ${ }^{9}$ acetone and acetoacetic acid, ${ }^{10}$ etc.

II. Combinations of the above conditions producing varied reduction products of the picric acid, some of which are colorless. ${ }^{11}$

12. Errors resulting from the action of light. ${ }^{12}$

SHATILE, WASH.

${ }_{1}$ Folin, Z. physiol. Chem., 4I, 223 (1904); Am. J. Physiol., 13, 45 (1905); J. Biol. Chem., 17, 463 (1914); see also Jaffe, $Z$. physiol. Chem., I0, 391 (1886).

2 Long, This Journal, 38, 716 (Igi6).

3 Taylor, J. Biol. Chem., 9, I9 (I9Ir).

- Since 50 volumes of water are used in Folin's method, this effect may become very pronounced, especially if very unequal depths of solutions are matched. See Cook, Thens Joursal, 3 I, 678 (1909); Smith and Meyers, Proc. A. O. A. C., 1909; U. S. Dept. Agr. Bur., Bull. I32, 160.

s Mellanby, J. Physiol., 36, 447 (1908); Chapman, Btit. Med. J., Dec. 12, 1908; Chem. News, 100, 175 (1909); A nalyst, 34, 475 (1910). A temperature of $20^{\circ}$ is recommended by Chapman.

- Standing for 5 minutes is recommended by Folin and also by Cook, This Journal, 31, 685 (1909).

${ }^{7}$ Helmer, Pharm. J., 78, 683 (1907); Emmett and Grindley, J. Biol. Chem., 2, 309 (1906); 3, 49 I (1907); Cook, Thrs Journal, 3I, 677 (rgog).

S Cook, Loc. cit.

- Taylor, J. Biol. Chem., 9, 19 (Igr I); Rona, Biochem. Z., 27, 348 (I911).

${ }^{10}$ Krause, Proc. Physiol. Soc., 1914; J. Physiol., 48, 41, 55 (1914); Cathcart and Orr, Proc. Physiol. Soc., I914; J. Physiol., 48, 21, 22 (1914); J. Chem. Soc., 106, 505 (1914); Morris, J. Biol. Chem., 21, zor (1915).

" Chapman, loc. cit.; also TuIs Journal, 36, 404 (1914).

12 Hunter and Campbell, J. Biol. Chem., 28, 335 (1916). 